\title{
Modelagem de Sistemas VoIP: Análise de Características da Sessão
}

\author{
Carlos Marcelo Pedroso, Eduardo Parente Ribeiro e Edgard Massahiro Munetiko
}

\begin{abstract}
Resumo-Neste trabalho serão apresentados modelos que podem descrever o comportamento de sessões de conversação que utilizam tecnologias de transmissão de voz sobre o protocolo IP. Foram analisados dados obtidos em duas grandes operadoras de telecomunicações brasileiras e avaliadas duas métricas relacionadas à dinâmica do comportamento das sessões: o processo de chegada e o tempo de serviço.

Palavras-Chave-VoIP, modelos de tráfego.

Abstract-This work introduces models that can describe the behavior of Voice over IP sessions. We used data from two major Brazilian telecommunications operators and examine two metrics related to the dynamic behavior of the sessions: the inter-arrival and service times.
\end{abstract}

Keywords - VoIP, traffic models.

\section{InTRODUÇÃO}

O uso de tecnologias de transmissão de voz sobre IP (VoIP, Voice over IP) está aumentando entre as operadoras de telecomunicações. Pode-se observar duas situações de emprego da tecnologia: (a) operadoras oferecendo serviços de VoIP "puros", onde o usuário origina/recebe chamadas utilizando protocolos VoIP; e (b) operadoras que fazem a conversão do tráfego gerado em outras tecnologias para padrões VoIP, facilitando o transporte dentro da rede da operadora.

Por outro lado, a maioria dos estudos diponíveis sobre sistemas VoIP reflete a situação particular de uma empresa ou universidade, como em [1]-[3]. Em Chen et al. [4] foi realizada a modelagem do tempo de duração de chamadas em uma operadora comercial de telefonia celular VoIP de Taiwan sobre uma amostra de 20000 chamadas. Foi verificada a característica de cauda longa no histograma dos dados. Foi ajustada uma mistura de duas distribuições lognormal aos dados.

Neste artigo serão apresentados os modelos que caracterizam o comportamento das chamadas (ou sessões) utilizandose amostras obtidas em duas grandes operadoras de telefonia no Brasil. As métricas de desempenho em estudo são: (a) o tempo de duração e (b) o intervalo de tempo entre sessões. O objetivo é produzir modelos representativos que possam ser utilizados para o estudo de desempenho de tais sistemas. A primeira operadora oferece um serviço VoIP "puro" e a segunda gera as chamadas em uma rede de telefonia celular, mas realiza o transporte através de um sistema VoIP; em ambos os casos, o backbone da rede utiliza uma rede Ethernet

Carlos Marcelo Pedroso é Professor na Universidade Federal do Paraná e na Pontifícia Universidade Católica do Paraná.

Eduardo Parente Ribeiro é Professor na Universidade Federal do Paraná.

Edgard Massahiro Munetiko é estudante do curso de Pós Graduação em Engenharia Elétrica da Universidade Federal do Paraná. não congestionada. Em [5] foram analisadas as principais características do tráfego RTP agregado: intervalo de tempo entre pacotes, tamanho do pacote, codecs utilizados e tráfego total para dados da primeira operadora coletados nos anos de 2006 e 2007.

Este artigo está estruturado da seguinte forma. A seção II mostra os principais modelos para caracterização de tráfego de voz em uso atualmente. A seção III descreve o conjunto de dados em estudo, e a seção IV apresenta a modelagem das métricas em estudo. Finalmente, os resultados são discutidos na seção $\mathrm{V}$ e as conclusões e trabalhos futuros são apresentados na seção VI.

\section{Conceitos Fundamentais}

Nesta seção serão apresentados os conceitos fundamentais que foram utilizados neste trabalho para modelagem do tempo de duração da sessão e do intervalo de tempo entre sessões.

\section{A. Distribuições de cauda pesada}

Uma variável aleatória $X$ possui distribuição de cauda pesada se [6]:

$$
\operatorname{Pr}\{X>x\} \sim c . x^{-\alpha}, \quad x \rightarrow \infty
$$

onde $\alpha$ é chamado parâmetro de forma e $c$ é uma constante positiva. Distribuições de cauda pesada possuem uma série de propriedades que as diferenciam das distribuições mais comuns (como a distribuição exponencial ou normal). A principal característica de uma variável aleatória com estas características é a variabilidade extrema exibida.

A distribuição de Pareto é uma distribuição de cauda pesada cuja função de distribuição cumulativa é dada pela Equação 2 [6],

$$
\operatorname{Pr}\{X \leq x\}=1-\left(\frac{\beta}{x}\right)^{\alpha}, \quad x>0, \alpha>0
$$

onde $\alpha$ é o parâmetro de forma e $\beta$ é chamado de parâmetro de localização. A esperança é dada por $E[X]=\alpha \beta /(\alpha-$ $1), \alpha>1$. Para $1<\alpha \leq 2$, a distribuição apresenta variância não convergente.

Conforme $\alpha$ diminui, uma maior quantidade de valores estarão contidos na cauda da distribuição; ou seja, a probabilidade de se encontrar valores muito grandes entre os dados aumenta. 


\section{B. Auto-similaridade}

Um processo é dito auto-similar do ponto de vista estocástico se mantém suas características estocásticas sobre uma certa faixa de escalas, de modo a satisfazer a relação dada pela Equação 3. Esta relação indica que um processo dado por $X_{m t}$ apresenta as mesmas características em termos de distribuição de probabilidade (indicada pelo símbolo $\cong_{d}$ ) de um processo $X_{t}$ re-escalonado por um fator $m$ e multiplicado pelo fator $m^{H}$.

$$
X_{m t} \cong_{d} m^{H} X_{t}, m>0
$$

Uma série é auto-similar se $0,5<H \leq 1,0$. A autosimilaridade foi reportada em inúmeros casos no tráfego de telecomunicaçoes, como apresentado por [7]-[10], entre muitos outros.

Uma das explicações sobre as causas auto-similaridade do tráfego de redes é a natureza dos objetos transmitidos na Internet: tamanho de arquivos, intervalos de tempo entre requisições ou mesmo tempo que o usuário processa informações são comumente descritos por distribuições de cauda pesada [11] [7], que torna o tráfego total observado uma série temporal auto-similar.

\section{Modelo ON-OFF}

Um dos modelos mais populares para geração de tráfego de voz é o modelo $O N-O F F$ [12]. Neste modelo, os pacotes são gerados somente durante o período onde o estado do sistema é $O N$. Neste estado, os pacotes são gerados com intervalos fixos entre chegadas. A sucessão entre estados $O N$ e $O F F$ é modelado pela distribuição exponencial.

O modelo IPP (Interrupted Poisson Process) modifica o modelo $O N-O F F$ de tal maneira que, quando o processo está no estado $O N$, a chegada dos pacotes será realizada de acordo com a distribuição de Poisson.

1) Modelo ON-OFF com distribuição de cauda longa: Neste modelo, cada fluxo tem um comportamento ON-OFF, onde o tempo que o fluxo permanece $O N$ ou $O F F$ possui uma distribuição de cauda pesada, como a distribuição de Pareto [6]. O tráfego agregado resultante da soma de diversos fluxos apresenta comportamento assintoticamente auto-similar. Este modelo tem sido freqüentemente utilizado para produção de tráfego em simulações.

$\mathrm{O}$ tráfego produzido pelo modelo $\mathrm{ON}-\mathrm{OFF}$, com o tempo de permanência em um dos dois estados modelado por uma distribuição de cauda pesada, possui características autosimilares para $\alpha \leq 2$ de acordo com a relação apresentada pela Equação 4 [13] [14].

$$
H=\frac{3-\alpha}{2}
$$

Um dos métodos utilizados para verificar se uma distribuição possui cauda pesada é através da análise da distribuição complementar em escala logarítmica (log-log complementary distribution, LLCD). Deve ser observado o gráfico que representa o valor da distribuição de probabilidade acumulada complementar $\bar{F}(x)=1-F(x) \operatorname{com} F(x)=$ $\operatorname{Pr}\{X<=x\}$, em escala logarítmica, de modo a procurar a invariância apresentada na Equação 5, para valores grandes de $x$. Na prática, escolhe-se um valor $x_{0}$ a partir do qual o gráfico passa a ser linear, ajustando uma reta e estimando o valor de $\alpha$ através do coeficiente angular. Este método foi utilizado neste trabalho.

$$
\frac{d \log (\bar{F}(x))}{d \log (x)}=-\alpha
$$

\section{Conjunto DE DAdos UTILIZADO}

O conjunto de dados em estudo refere-se ao tráfego coletado em duas grandes operadoras de telecomunicações no Brasil. Em ambos os casos o tráfego foi coletado no backbone da rede. Os detalhes sobre as tecnologias utilizadas em cada operadora serão descritos a seguir.

\section{A. Operadora 1}

A operadora 1 fornece um serviço de Voz sobre IP utilizando o protocolo SIP (Session Initiation Protocol [15]) para sinalização, com os fluxos de voz transportados pelo protocolo RTP (Real Time Protocol [16]). O SIP é utilizado para estabelecer chamadas através do protocolo IP, atua na camada de aplicação e é um padrão da Internet Engineering Task Force (IETF).

O SIP foi projetado para interagir com outros protocolos da internet, iniciando, modificando e terminando a sessões, independente do tipo de mídia ou aplicação em uso. O protocolo caracteriza-se como simples, flexível, estável, baseado em texto, e independente do protocolo de transporte. Uma vez iniciada a conferência, a voz é codificada/decodificada por um codec e transmitida através do protocolo RTP.

A rede analisada possuía aproximadamente 10.000 usuários na época da coleta do tráfego em setembro de 2007. Em termos gerais, a rede de acesso é formada por enlaces ADSL (Asymmetric Digital Subscriber Line). O tráfego VoIP gerado pelos usuários é direcionado para uma rede Ethernet não congestionada. Para a coleta de dados, diversas portas do switch Ethernet que serve como backbone da rede foram espelhadas, de modo que o tráfego total do sistema VoIP foi capturado utilizando-se o analisador de protocolos Open Source Wireshark [17]. O sistema também é integrado com o sistema de telefonia convencional através de um media gateway, que permite a realização de chamadas com a rede pública de telefonia comutada.

Neste trabalho foram analisados o tempo de duração das sessões e o intervalo de tempo entre sessões. Para isto, foram identificadas as mensagens INVITE, ACK e BYE do protocolo SIP - o proceso de inicialização da chamada é ilustrado na Figura 1. É importante enfatizar que foram apenas consideradas as sessões que terminaram com uma mensagem BYE. Toda a sinalização é intermediada através de um servidor Proxy, que não é representado na Figura 1. Além disso, a mensagem BYE pode ser originada em qualquer um dos agentes de usuários.

\section{B. Operadora 2}

A operadora 2 fornece serviços de telefonia móvel. O tráfego de voz destinado à outras operadoras de telefonia 


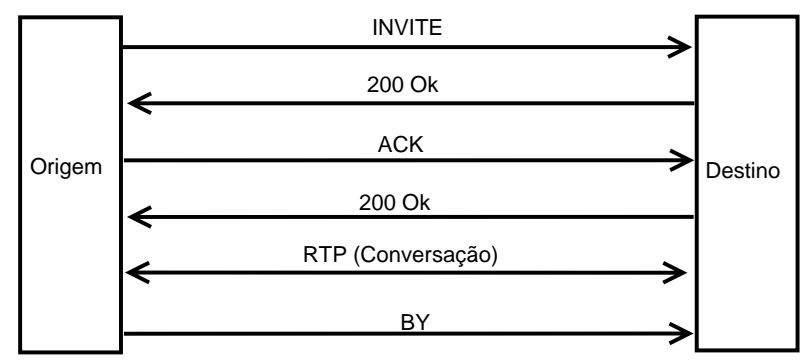

Fig. 1. Sequência das principais mensagens que iniciam uma chamada com protocolo SIP

TABELA I

SUMÁRIO DE ESTATÍSTICAS BÁSICAS DOS DADOS EM ESTUDO

\begin{tabular}{lllll}
\hline & Data & Tempo & $\widehat{\tau}$ & $\widehat{\delta}$ \\
\hline Operadora 1 & $20 / 09 / 2007$ & 8h45min a 12h50min & 1,404 & 120,02 \\
Operadora 2 & $14 / 04 / 2009$ & 8h00min a 16h00min & 1,336 & 62,11 \\
onde $\widehat{\delta}$ representa o tempo médio de duração da sessão.e $\widehat{\tau}$ representa o \\
\multicolumn{4}{c}{ intervalo médio entre chegadas de sessões, ambos em segundos. }
\end{tabular}

(móvel e fixo) é encaminhado através de media gateways que estão conectados ao um switch ethernet formando uma rede local. As centrais celulares utilizam o protocolo BICC (Bearer Independent Call Control) [18] para sinalização. O sinal de voz codificado em AMR2 (Adaptive Multi-Rate compression) é transportado pelo protocolo RTP.

O diagrama simplificado para início e encerramento de seção é mostrado na Figura 2. Foram identificadas as mensagens IAM (Initial Address Message) e RLC (Release Complete message) do protocolo BICC. O intervalo entre sessões foi considerado o tempo entre mensagens IAM. A duração da sessão foi considerada como o tempo entre a mensagem IAM e a mensagem RLC. Foram desconsideradas sessões que não apresentavam a mensagem RLC.

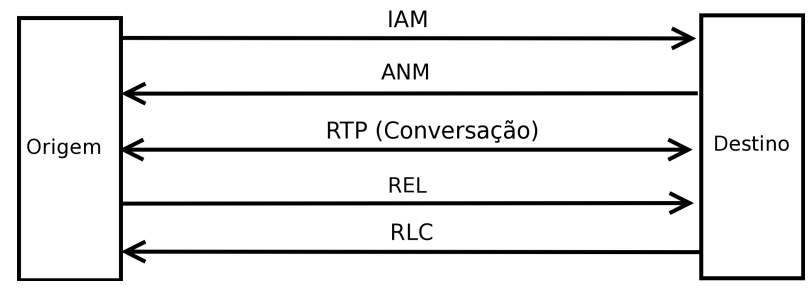

Fig. 2. Sequência simplificada de mensagens do protocolo BICC para estabelecimento e desconexão de chamadas

O tráfego considerado consiste de chamadas entre os clientes da operadora 2 e números de outras operadoras. Os dados foram coletados durante um período de $8 \mathrm{~h}$ ( 8 as 16h) em um dia útil de abril de 2009. Cerca de $80 \%$ dos usuários possuem plano de tarifação pré-pago e o restante tem plano pós-pagos por minutos ou com franquia.

\section{Modelagem das Sessões VoIP}

Nesta seção serão apresentados os modelos identificados para caracterizar o processo de chegada e o tempo de duração da sessão, de acordo com os dados empíricos observados.

\section{A. Processo de Chegada}

Para caracterizar o processo de chegada foram amostrados os intervalos de tempo entre o início de uma sessão e o início da sessão imediatamente subsequente. A taxa de chegada de sessões é calculada pelo inverso do intervalo de tempo entre o início de sessões. Para ambos os casos, a taxa de chegada caracteriza-se por um processo estacionário, ou seja, nenhum tipo de tendência pode ser observada dentro do intervalo de tempo onde foram coletadas as amostras.

Foi observada uma boa aderência da distribuição acumulada de probabilidade do intervalo entre chegadas amostrado nas duas operadoras à distribuição teórica exponencial, dada por $\operatorname{Pr}\{X<=x\}==F(x)=1-e^{-\lambda x}$, onde $x$ representa o intervalo de tempo entre chegadas. A realização do teste de aderência Kolmogorov-Smirnov indica que esta hipótese não pode ser descartada para os dados de ambas operadoras. Tomando-se um conjunto de 1 hora de dados em qualquer uma das duas amostras o valor $p$ obtido tipicamente é da ordem de 0,8 ; para rejeitar a hipótese, o valor deveria estar abaixo de 0,05 [19].

Também foi realizado o teste sobre a estacionariedade da série utilizando a função de autocorrelação de ambas as amostras e o resultado indica que o intervalo de tempo de chegadas é independente em relação ao deslocamento (ou lag). Desta forma, este é um processo Markoviano; este resultado era esperado e confirma as expectativas.

\section{B. Processo de Atendimento}

Para estudar o processo de atendimento foram analisados os tempos de duração da sessão, onde foram detectadas características de cauda pesada através da análise do gráfico da distribuição complementar em escala logarítimica, conforme apresentado anteriormente na seção II. A Figura 3 mostra o gráfico da distribuição complementar em escala logarítmica para o tempo de duração da sessão amostrado nas duas operadoras.

Para os dados da Operadora 1 (Figura 3, esquerda), é possível observar que a distribuição complementar comportase como uma reta na escala logarítmica a partir de do tempo de duração da sessão de aproximadamente 100 segundos, o que indica a presença da cauda pesada. Graficamente pode ser constatado um ângulo de aproximadamente $-63^{\circ}$, o que pode ser utilizado de acordo com a Equação 5 para estimar o valor do parâmetro de forma $\widehat{\alpha} \simeq 1,96$.

Para a Operadora 2 também é apresentado o gráfico da distribuição complementar em escala logarítimica (Figura 3, direita), onde observa-se que podem ser diferenciados dois regimes: 1) para tempos de duração da sessão de 100 até em torno de 1200 segundos e 2) para tempos maiores que 1200 segundos. Para o regime 1, pode ser ajustada uma reta que possui ângulo de aproximadamente $-53^{\circ}$, o que leva a $\widehat{\alpha} \simeq 1,33$. No entanto, o regime 2 mostra que o tempo de duração da sessão perde sua característica de cauda longa e converge rapidamente, com $\widehat{\alpha} \simeq 4,7$. A identificação destes dois regimes sugere o ajuste de parâmetros diferenciados para cada um dos regimes. No entanto, neste trabalho não 

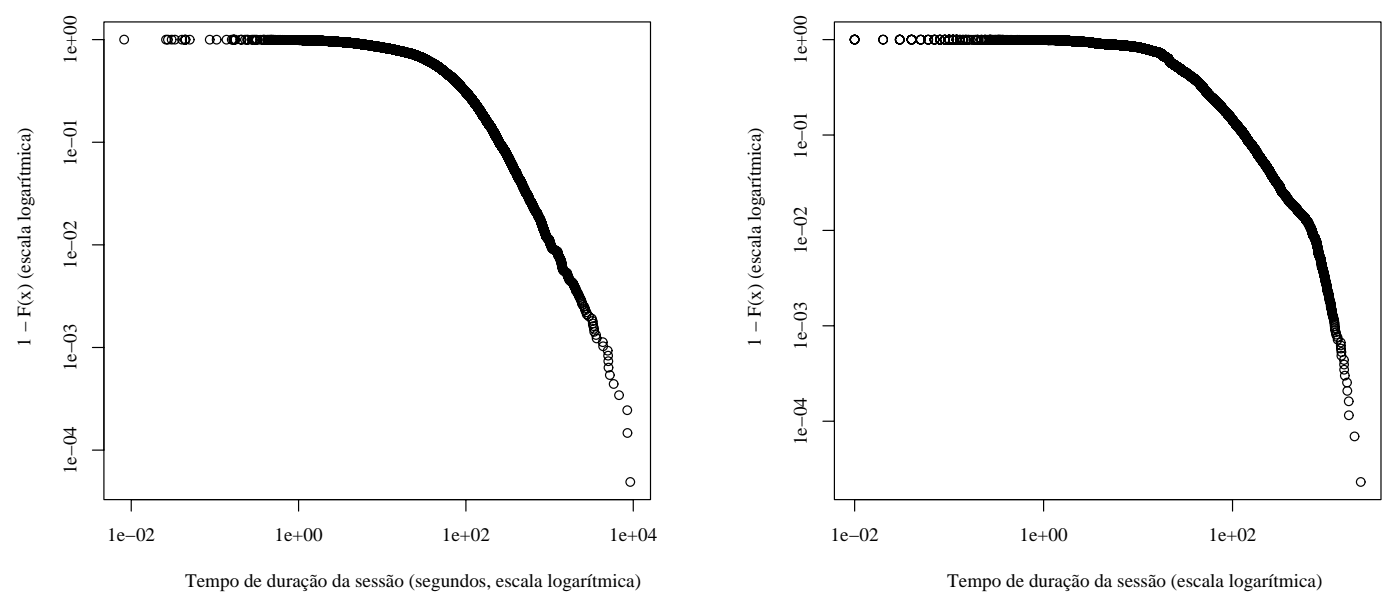

Fig. 3. Distribuição complementar em escala logarítmica para o tempo de duração da sessão. À esquerda, dados da Operadora 1. À direita, dados da Operadora 2.
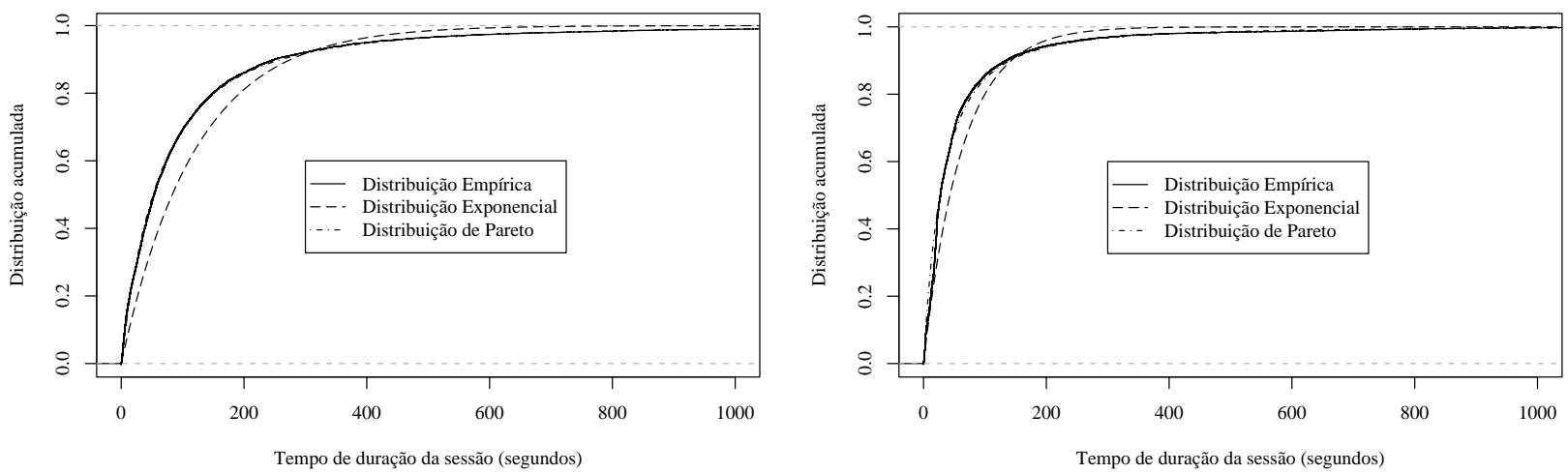

Fig. 4. Função de distribuição acumulada de probabilidade do tempo de duração da sessão, comparada com diversas distribuições teóricas. À esquerda, dados da Operadora 1. À direita, dados da Operadora 2.
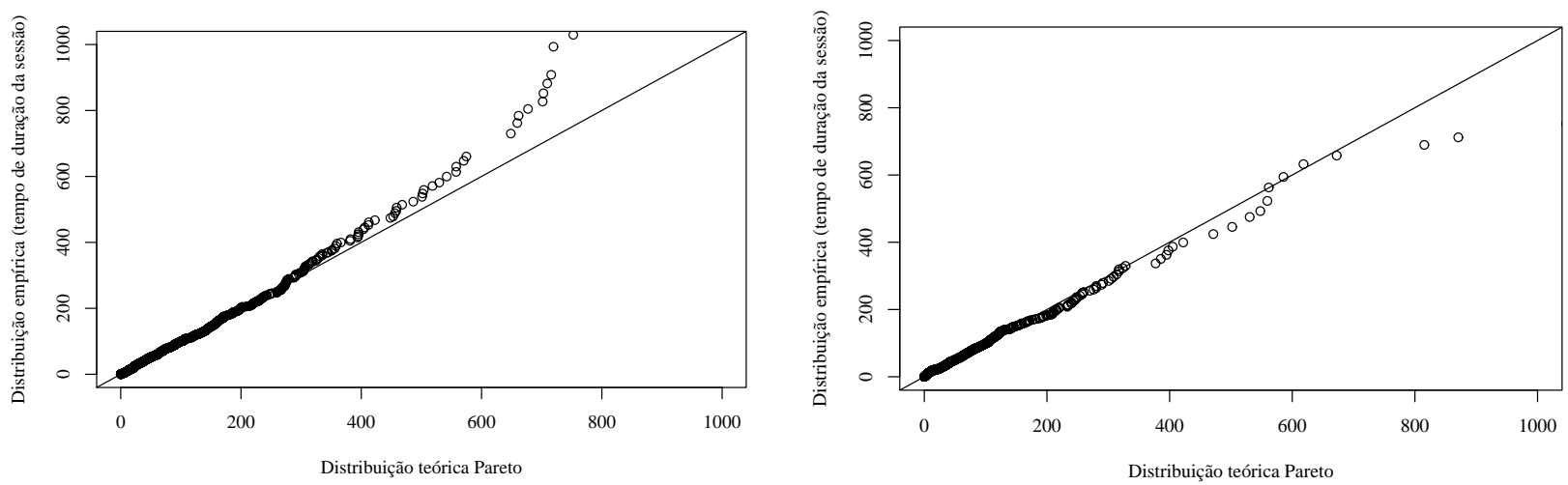

Fig. 5. Gráfico Quantile-Quantile (QQ-Plot) da probabilidade do tempo de duração da sessão, comparada com a distribuição teórica de Pareto. À esquerda, dados da Operadora 1. À direita, dados da Operadora 2. 
XXVI SIMPÓSIO BRASILEIRO DE TELECOMUNICAÇÕES - SBrT’09, 29 DE SETEMBRO A 2 DE OUTUBRO DE 2009, BLUMENAU, SC

TABELA II

PARÂMETRos estimados PARA Distribuição de PARETo PARA O TEMPO DE DURAÇÃO DA SESSÃO

\begin{tabular}{lll}
\hline & Forma $(\widehat{\alpha})$ & Escala $(\widehat{\beta})$ \\
\hline Operadora 1 & 2.16 & 63.42 \\
Operadora 2 & 1.95 & 32.05
\end{tabular}

será utilizado este recurso porque tornaria mais complexo o estabelecimento de mecanismos tratáveis para análise.

Também foram estimados os parâmetros da distribuição de Pareto (Equação 2) para os dados de ambas as operadoras utilizando-se o método desenvolvido por [20] e implementado no software estatístico $\mathrm{R}$ [21], com os parâmetros estimados para a distribuição de Pareto apresentados na Tabela II. A Figura 4 mostra a distribuição acumulada de probabilidade do tempo de duração da sessão comparada com a distribuição de Pareto. Nesta figura também é apresentada a curva para distribuição exponencial, para efeito de comparação por ser esta uma distribuição que converge rapidamente. A comparação da distribuição empírica (linha contínua) com a distribuição teórica exponencial evidencia o comportamento de cauda pesada, onde pode perceber-se a lenta convergência da distribuição empírica se comparada à rápida convergência exibida pela distribuição exponencial. Também é mostrada a distribuição teórica de Pareto, com os parâmetros estimados. Observa-se para os dados da Operadora 1 (Figura 4, esquerda) uma boa aderência à distribuição de Pareto, onde o leitor deve observar a figura com atenção para identificar a linha pontilhada representando a distribuição teórica, que está praticamente sobreposta à linha contínua que representa a distribuição empírica. Para os dados da Operadora 2 (Figura 4direita) também pode ser observada uma boa aderência à distribuição de Pareto.

Para comprovar a aderência dos dados ao modelo, é apresentado na Figura 5 o gráfico quantile-quantile ( $Q Q-P l o t)$, que mostra os quantiles da distribuição empírica em relação aos quantiles da distribuição teórica de Pareto com os parâmetros estimados. O QQ-Plot é utilizado para determinar graficamente se dois conjuntos de dados possuem a mesma distribuição de probabilidade. Quando os dois conjuntos possuem a mesma distribuição, o resultado é que os pontos (representados por círculos) são colocados sobre a linha contínua mostrada na Figura 5. Para os dados da Operadora 1 (Figura 5, esquerda), o QQ-Plot mostra uma boa aderência à distribuição de Pareto, com os dados empíricos apresentando um número maior de valores extremos em relação ao que seria previsto pela distribuição teórica. Na Figura 5, direita, é apresentado o QQPlot para os dados da Operadora 2, onde é constatada uma boa aderência dos dados à distribuição de Pareto.

\section{AnÁlise de Resultados e Discuss Ão}

Para a análise de resultados, é importante lembrar que a Operadora 1 oferece um serviço de Voz sobre IP onde os usuários utilizam terminais VoIP na origem. A Operadora 2 não oferece um serviço de $\mathrm{Voz}$ sobre IP diretamente ao usuário; a rede analisada refere-se ao tráfego de telefonia celular que foi digitalizado e transportado com padrões VoIP dentro da rede da Operadora.

\section{A. Operadora 1}

Os resultados apresentados para a Operadora 1 indicam que podem ser utilizados os seguintes modelos para caracterizar o comportamento das sessões:

- Intervalo de tempo entre sessões: Processo Markoviano, caracterizado por uma distribuição exponencial;

- Tempo de duração da sessão: Caracterizado através de uma distribuição de cauda pesada com parâmetro de forma $\alpha \sim 2$, com os dados empíricos apresentando uma boa aderência à distribuição de Pareto.

Este resultado indica que a probabilidade de ocorrência de sessões com um tempo de duração muito grande não é desprezível. O valor encontrado para o parâmetro de forma indica que a variância do processo está no limite de convergência (ver seção II).

Como consequência, o estudo de desempenho do sistema não pode ser realizado utlizando-se modelos clássicos de filas (ex. sistema M/M/1 ou M/M/c). Poderia considerar-se o uso do modelo M/G/1 [19], desenvolvido por Pollaczek-Khinchin, no entanto isto não é possível devido à situação limite para o valor de $\alpha$ : o modelo $\mathrm{M} / \mathrm{G} / 1$ necessita que a variância do processo seja convergente. Um modelo factível seria um modelo de filas $\mathrm{M} / \mathrm{P} / 1$ (onde $\mathrm{P}$ indica o tempo de atendimento modelado pela distribuição de Pareto), mas os modelos conhecidos não apresentam resultados analíticos para $\alpha \leq 2$ [22], [23]. Um método possível para o estudo de desempenho de tal sistema é através do uso de simulações computacionais, embora o valor de $\alpha$ no limite para convergência da variância também dificulte a análise dos resultados. Um sistem M/P/1 apresenta tempos médios de fila muito maiores do que aqueles que seriam previstos com o modelo $\mathrm{M} / \mathrm{M} / 1$ [6].

O comportamento do tempo de duração da sessão está intimamente ligado ao modelo de tarifação em uso. Em sistemas VoIP como o estudado, em ligações entre terminais VoIP o tempo de duração da sessão não representa custo adicional ao usuário. Isto significa que a sessão não será encerrada em função do custo da ligação, o que gera o comportamento observado.

\section{B. Operadora 2}

A análise dos dados da Operadora 2 indicou que os seguintes modelos podem ser utilizados para caracterizar o comportamento das sessões:

- Intervalo de tempo entre sessões: Disribuição exponencial, como no caso da Operadora 1;

- Tempo de duração da sessão: Foi caracterizado com a distribuição de Pareto - no entanto, a partir de 1200 segundos a distribuição converge rapidamente.

A distribuição de probabilidade acumulada do tempo de duração da sessão observado apresentou uma lenta convergência, que impede o uso de distribuições de probabilidade tradicionais (por ex. distribuição exponencial) para caracterizar o processo. 
O tempo médio de duração da sessão observado nesta operadora é igual a metade do tempo médio de duração da sessão observado na Operadora 1. A diferença de comportamento para o tempo de duração da sessão observado na Operadora 1 e Operadora 2 pode ser explicada através do sistema de tarifação utilizado pela Operadora 2. O tráfego analisado é tarifado de diversas maneiras, incluindo o sistema pré-pago e pós-pago, com diversos tipos de planos e descontos sendo oferecidos aos assinantes. Os resultados permitem concluir que o sistema de tarifação de telefonia celular utilizado pela Operadora 2 está favorecendo a ocorrência de tempos maiores de sessão do que a observada em um sistema de tarifação tradicional.

O estudo de desempenho do sistema pode ser realizado utilizando-se uma distribuição de Pareto truncada, como descrito por [22], utilizando-se simulações computacionais.

\section{CONClusões E TRABALhos Futuros}

Os resultados obtidos permitem concluir que os sistemas em estudo podem ser caracterizados através de um modelo ON-OFF, onde o tempo de permanência no estado ON segue uma distribuição de cauda pesada (foi utilizada distribuição de Pareto) e o estado OFF segue uma distribuição exponencial. A possível causa para o comportamento de cauda pesada para a distribuição de probabilidade do tempo da sessão é o regime de tarifação, o que pode ser evidenciado na comparação entre as características observadas nas duas operadoras. A Operadora 2 utiliza um sistema de tarifação mais rígido se comparado com a Operadora 1, uma vez as chamadas são originadas no sistema de telefonia celular, e a duração da chamada tende a ser menor. No entanto, ambos os processos mostram características de grande variância.

A maneira mais adequada disponível para a realização de estudos sobre sistemas com estas características é através de simulações, uma vez que existem problemas ainda não resolvidos para o estudo analítico de sistemas com filas onde o tempo de serviço segue uma distribuição de cauda pesada. Neste trabalho foram apresentadas as características do comportamento das sessões que permitem a realização de tais simulações.

Como trabalho futuro, dentro do estado $\mathrm{ON}$, propomos o uso de um modelo ON-OFF, que serão chamados ACTIVEON e ACTIVE-OFF. Os tempos ACTIVE-ON e ACTIVEOFF dependem fortemente do tipo de codec utilizado. Foi mostrado anteriormente em [5] que no sistema da Operadora 1 são utilizados diversos codecs de forma que o comportamento dos tempos ACTIVE-ON e ACTIVE-OFF necessitam ser caracterizados para o estabelecimento de modelos. Como consequiência, espera-se obter um modelo representativo para geração de tráfego VoIP para simulações computacionais. Este estudo está em andamento.

Os dados disponibilizados neste artigo podem ser utilizados em uma série de estudos, como planejamento de capacidade, sistemas de tarifação, controle de admissão, entre outros.

\section{REFERÊNCIAS}

[1] W.Jiang and H.Schulzrinne, "Analysis of on-off patterns in voip and their effect on voice traffic aggregation," in Proceedings of 9th IEEE International Conference on Computer Communication Networks, 2000.

[2] B.Bellalta, M.Oliver, and D.Rincn, "Capacity and traffic analysis of voip services over gprs mobile networks," in report TIC2001-0956-C04. Technical University of Catalonia, University Pompeu Fabra, Spain., 2001.

[3] E.Casilari, H.Montes, and F.Sandoval, "Modelling of voice traffic over ip networks," in Proc. of Communication Systems, Networks and Digital Signal Processsing (CSNDSP), 2002.

[4] W.-E. Chen, H.-N. Hung, and Y.-B. Lin, "Modeling voip call holding times for telecommunications," Network, IEEE, vol. 21, no. 6, pp. 2228, November-December 2007.

[5] C. M. Pedroso, J. Caldeira, and K. Fonseca, "Análise da evolução de características de tráfego VoIP," in XXVI Simpósio da Sociedade Brasileira de Telecomunicações, 2008.

[6] W. Willinger and K. Park, Self-similar network traffic and performance evaluation, 1st ed. New York: John Wiley \& Sons, 2000.

[7] W. Leland, M. Qaqqu, W. Willinguer, and D. Wilson, "On the selfsimilar nature of Ethernet traffic (extended version)," IEEE/ACM Transactions on Networking, vol. 2, no. 1, pp. 1-15, February 1994.

[8] M. W. Garrett and W. Willinger, "Analysis, modeling and generation of self-similar vbr video traffic," in SIGCOMM '94: Proceedings of the conference on Communications architectures, protocols and applications. New York, NY, USA: ACM Press, 1994, pp. 269-280.

[9] A. Veres, Z. Kenesi, S. Molnár, and G. Vattay, "TCP's role in the propagation of self-similarity in the internet." Computer Communications, vol. 26, no. 8, pp. 899-913, 2003.

[10] W.-B. Gong, Y. Liu, V. Misra, and D. F. Towsley, "Self-similarity and long range dependence on the internet: a second look at the evidence, origins and implications ." Computer Networks, vol. 48, no. 3, pp. 377399, 2005.

[11] M. Crovella and A. Bestavros, "Self-similarity in World Wide Web traffic: Evidence and possible causes," IEEE/ACM Transactions on Networking, vol. 5, no. 6, pp. 835-846, 1995.

[12] A. Adas, "Traffic models in broadband networks," IEEE Communications Magazine, vol. 37, no. 7, pp. 82-89, July 1997.

[13] M. E. Crovella, M. S. Taqqu, and A. Bestavros, "Heavy-tailed probability distributions in the World Wide Web," in A Practical Guide To Heavy Tails, R. J. Adler, R. E. Feldman, and M. S. Taqqu, Eds. New York: Chapman and Hall, 1998, ch. 1, pp. 3-26. [Online]. Available: http://www.cs.bu.edu/faculty/crovella/paper-archive/web-tails.ps

[14] M. Crovella, M. Taqqu, and A. Bestavros, A Practical Guide to Heavy Tail. Chapman \& Hall, 1998, ch. 1, pp. 3-26.

[15] J. Rosenberg, H. Schulzrinne, G. Camarillo, A. Johnston, J. Peterson, R. Sparks, M. Handley, and E. Schooler, "RFC3261: Session initiation protocol," 2002.

[16] H. Schulzrinne, S. Casner, R. Frederick, and V. Jacobson, "STD 64: RTP: A transport protocol for real-time applications," Jul. 2003.

[17] A. Orebaugh, G. Ramirez, J. Burke, and L. Pesce, Wireshark \& Ethereal Network Protocol Analyzer Toolkit (Jay Beale's Open Source Security). Syngress Publishing, 2006.

[18] ITU-T, "Q.1901 bearer independent call control protocol," Series Q: switching and signalling, 2000.

[19] R. Jain, The art of computer systems performance analysis: techniques for experimental design, measurement, simulation and modeling. John Wiley \& Sons, 1991.

[20] J. R. M. Hosking and J. F. Wallis, "Parameter and quantile estimation for the generalized pareto distribution," Technometrics, vol. 29, no. 3, pp. 339-349, 1987.

[21] R Development Core Team, R: A Language and Environment for Statistical Computing, R Foundation for Statistical Computing, Vienna, Austria, 2005, ISBN 3-900051-07-0. [Online]. Available: http://www.R-project.org

[22] E. Yucesan, C. H. Chen, J. L. Snowdon, J. M. Charnes, D. Gross, and J. F. Shortle, "Difficulties in simulating queues with pareto service," in Simulation Conference, 2002.

[23] P. Argibay-Losada, A. Suarez-Gonzalez, C. Lopez-Garcia, R. RodriguezRubio, J. Lopez-Ardao, and D. Teujeiro-Ruiz, "On the simulation of queues with pareto service," in European Simulation and Modelling Conference (ESM), 2003. 